\title{
Patient-Controlled Nutrition After Abdominal Surgery: Novel Concept Contrary to Surgical Dogma
}

\author{
Hyung Ook Kim, Mingoo Kang*, Sung Ryol Lee, Kyung Uk Jung, Hungdai Kim, Ho-Kyung Chun \\ Department of Surgery, Kangbuk Samsung Hospital, Sungkyunkwan University School of Medicine, Seoul, Korea
}

Purpose: According to surgical dogma, patients who are recovering from general anesthesia after abdominal surgery should begin with a clear liquid diet, progress to a full liquid diet and then to a soft diet before taking regular meals. We propose patient-controlled nutrition (PCN), which is a novel concept in postoperative nutrition after abdominal surgery. Methods: A retrospective pilot study was conducted to evaluate the feasibility and effects of PCN. This study was carried out with a total of 179 consecutive patients who underwent a laparoscopic appendectomy between August 2014 and July 2016. In the PCN group, diet was advanced depending on the choice of the patients themselves; in the traditional group, diet was progressively advanced to a full liquid or soft diet and then a regular diet as tolerated. The primary endpoints were time to tolerance of regular diet and postoperative hospital stay.

Results: Time to tolerance of a regular diet $(\mathrm{P}<0.001)$ and postoperative hospital stay $(\mathrm{P}<0.001)$ showed statistically significant differences between the groups. Multivariate analysis using linear regression showed that the traditional nutrition pattern was the only factor associated with postoperative hospital stay $(\mathrm{P}<0.001)$. Multivariate analysis using logistic regression showed that traditional nutrition was the only risk factor associated with prolonged postoperative hospital stay ( $\geq 3$ days).

Conclusion: After abdominal surgery, PCN may be a feasible and effective concept in postoperative nutrition. In our Early Recovery after Surgery program, our PCN concept may reduce the time to tolerance of a regular diet and shorten the postoperative hospital stay.

Keywords: Postoperative care; Diet; Nutritional support; Abdominal surgery; Appendectomy

\section{INTRODUCTION}

How long will surgical dogma prevail in perioperative manage-

Received: March 12, 2018 • Accepted: May 29, 2018

Correspondence to: Hyung Ook Kim, M.D.

Department of Surgery, Kangbuk Samsung Hospital, Sungkyunkwan

University School of Medicine, 29 Saemunan-ro, Jongno-gu, Seoul 03181, Korea

Tel: +82-2-2001-8550, Fax: +82-2-2001-2131

E-mail: ho115.kim@samsung.com

ORCID code: https://orcid.org/0000-0003-0603-8766

*Hyung Ook Kim and Mingoo Kang contributed equally as co-first authors. This paper was presented as a poster at 51th Korean Society of Coloproctology Annual Meeting, Gwangju, Korea, March 30-April 1, 2018.

(C) 2018 The Korean Society of Coloproctology

This is an open-access article distributed under the terms of the Creative Commons Attribution NonCommercial License (http://creativecommons.org/licenses/by-nc/4.0) which permits unrestricted non-

commercial use, distribution, and reproduction in any medium, provided the original work is properly cited ment without being subjected to strict clinical evidence? One piece of representative surgical dogma is the routine use of nasogastric tubes to decompress the gastrointestinal (GI) tract after abdominal surgery. Indeed, for more than half a century, surgeons have relied on nasogastric decompression after GI surgery to hasten recovery. However, a meta-analysis in 1995 and another in 2005 for the routine use of nasogastric tubes showed little evidence of benefit $[1,2]$. Moreover, those analyses showed that fever, atelectasis, and pneumonia were less common and bowel function was restored earlier in patients managed without nasogastric tubes $[1,2]$.

The initial choice and timing of diet after abdominal surgery have been debated, in addition to the time to advance to a regular diet, because GI motility is disrupted during convalescence. Many clinical studies have supported the idea that early oral feeding (EOF) following abdominal surgery is associated with accelerated patient recovery [3-5]. Furthermore, our previous study showed that patients have a high tolerability to EOF following laparo- 
scopic colorectal surgery without other supportive treatment [6]. Although surgeons are enthusiastic about EOF, most of them initially administer a clear liquid diet to patients after abdominal surgery and advance to a regular diet gradually because of deeprooted concern about the risk of postoperative nausea and vomiting [7]. Clinical signs and symptoms, physical examinations, and laboratory or radiologic findings are often used to predict the tolerance to EOF and to evaluate whether oral intake can be advanced to a regular diet. In patients not eligible for EOF, regular monitoring of bowel sounds and confirmation of flatulence and/ or bowel movements have been regarded as evidence that the patient is ready to advance their diet. However, clinical evidence that these actions are correlated with postoperative bowel function and integrity does not exist [8].

The most reliable indicator of tolerability to postoperative oral feeding is the subjective opinion of the patients themselves, and the choice of diet should depend on the patient's comfort and preference. Indeed, patients usually want to choose the time and type of diet, especially if surgical complications do not occur (in which case a nil per os diet might be required). Furthermore, no evidence exists that patient-selected postoperative diets lead to any more nausea or vomiting than the traditional progression of a clear liquid diet to a full liquid diet, to a soft diet, and finally to a regular diet [9].

Herein, we propose patient-controlled nutrition (PCN), which is a novel concept contrary to surgical dogma for postoperative nutrition after abdominal surgery. A retrospective pilot study was conducted with laparoscopic appendectomy patients who presented with simple appendicitis to evaluate the feasibility and effectiveness of PCN, as well as its effects on the postoperative outcomes following abdominal surgery.

\section{METHODS}

This study was carried out with 179 consecutive patients who presented with uncomplicated acute appendicitis and underwent a laparoscopic appendectomy by one of 2 surgeons between August 2014 and July 2016 at Kangbuk Samsung Hospital. Patients with complicated appendicitis, such as gangrenous or perforated appendicitis or appendicitis with the presence of a periappendiceal abscess, were excluded. The study was approved by the Ethics Committee at our institution (KBSMC 2017-02-013). The Institutional Review Board (IRB) waived the requirement for obtaining the informed consent.

All surgeries included in this study were performed by 1 of 2 experienced laparoscopic colorectal surgeons; the strategy for postoperative nutrition was decided based on surgeon preference. All patients were allowed a clear liquid diet after subjective full recovery from general anesthesia. Then, 1 surgeon advanced the diet depending on the choice of patients themselves (PCN group) within 3-12 hours according to the regular times that food was served at the hospital, and the other surgeon progressively ad- vanced the diet to full liquid or soft diet and then to regular diet, as tolerated (traditional group). Retrospective analyses were performed for both groups.

The laparoscopic appendectomy was performed using conventional 3-port laparoscopic techniques as previously described [10]. Using a modified Hasson technique to access the peritoneum, the surgeon inserted a 12-mm blunt laparoscopic port into the supraumbilical position, including the center of the umbilicus. Two additional 5-mm laparoscopic ports were inserted into the suprapubic and left iliac fossa positions under laparoscopic direct vision. The appendectomy was performed using common laparoscopic instruments including an ultrasonic shear (Harmonic Scalpel, Ethicon Endo-Surgery Inc., Cincinnati, OH, USA), a round loop (Laploop, Sejong Medical Co., Paju, Korea), and a bag (Lapbag; Sejong Medical Co.). The umbilical fascia was closed with 2-0 Vicryl sutures, and the subcutaneous layer was sutured with 4-0 Vicryl to the alignment of the skin edges without skin suture.

A urinary catheter was inserted in the operation room following general anesthesia. All anesthetic procedures were performed in a routine fashion. Intravenous patient-controlled analgesia was postoperatively employed for pain relief according to patient preference. The patient's diet was advanced based on surgeon preference. The urinary catheter was removed on the day of the operation or at $6 \mathrm{AM}$ on the first postoperative day according to patient preference. All patients were encouraged to ambulate on the first postoperative day.

Data were retrospectively collected after the study protocol had been approved by the Ethics Committee at our institute. The primary endpoints were time to tolerance of regular diet and postoperative hospital stay. Collected data included patient demographics, clinicopathologic characteristics, and perioperative outcomes including operative time, time to tolerance of regular diet, and length of hospital stay.

Results are expressed as means \pm standard deviations. Continuous variables were compared using the Student t-test. Discrete variables were analyzed with the chi-square test or Fisher exact test. Multivariate analyses using linear regression or logistic regression were performed to determine the risk factors associated with prolonged postoperative hospital stay. Statistical analyses were performed using a IBM SPSS Statistics ver. 19.0 (IBM Co., Armonk, NY, USA). P-values less than 0.05 were considered statistically significant.

\section{RESULTS}

In total, cases involving 179 patients with a mean age of $33.3 \pm$ 17.0 years were analyzed. Clinical data, including sex, body mass index (BMI), American Society of Anesthesiologists (ASA) physical status classification, history of previous major abdominal surgery, and perioperative outcomes are shown in Table 1. The outcomes were analyzed on an intention-to-treat basis.

Statistically significant differences in time to tolerance of a regu- 
Table 1. Clinicopathological characteristics and perioperative outcomes in the patient-controlled nutrition (PCN) group and the traditional group

\begin{tabular}{|c|c|c|c|}
\hline Characteristic & $\begin{array}{c}\text { PCN } \\
(n=84)\end{array}$ & $\begin{array}{l}\text { Traditional } \\
(\mathrm{n}=95)\end{array}$ & P-value \\
\hline Age (yr) & $31.1 \pm 15.0$ & $35.2 \pm 18.5$ & 0.108 \\
\hline Sex, male : female & $34: 50$ & $51: 44$ & 0.077 \\
\hline Body mass index $\left(\mathrm{kg} / \mathrm{m}^{2}\right)$ & $22.0 \pm 3.7$ & $22.8 \pm 3.6$ & 0.133 \\
\hline Underlying disease & $13(15.5)$ & $12(12.6)$ & 0.584 \\
\hline ASA PS classification & & & 1.000 \\
\hline 1 & $80(95.2)$ & $90(94.7)$ & \\
\hline$\|$ & $3(3.6)$ & $4(4.2)$ & \\
\hline III & $1(1.2)$ & $1(1.1)$ & \\
\hline IV & $0(0)$ & $0(0)$ & \\
\hline Preoperative WBC count (/mm³) & $11,879 \pm 4,962$ & $12,905 \pm 4,301$ & 0.140 \\
\hline $\begin{array}{l}\text { History of previous abdominal } \\
\text { surgery }\end{array}$ & $8(9.5)$ & $10(10.5)$ & 0.824 \\
\hline $\begin{array}{l}\text { Postoperative pain within } 24 \\
\text { hours (VAS) }\end{array}$ & $6.4 \pm 1.1$ & $6.3 \pm 0.9$ & 0.621 \\
\hline Postoperative opioid use & $35(41.7)$ & $45(47.4)$ & 0.444 \\
\hline Pathology, serosal inflammation & & & 0.288 \\
\hline No & $12(14.3)$ & $9(9.5)$ & \\
\hline Mild & $38(45.2)$ & $37(38.9)$ & \\
\hline Severe & $34(40.5)$ & $49(51.6)$ & \\
\hline Operation time (min) & $32.4 \pm 13.1$ & $39.2 \pm 16.1$ & 0.003 \\
\hline $\begin{array}{l}\text { Time to tolerance of regular diet } \\
\text { (hr) }\end{array}$ & $5.5 \pm 3.9$ & $18.3 \pm 3.8$ & $<0.001$ \\
\hline Postoperative hospital stay (day) & $1.83 \pm 0.8$ & $2.91 \pm 1.1$ & $<0.001$ \\
\hline
\end{tabular}

Values are presented as mean \pm standard deviation or number (\%).

ASA PS, American Society of Anesthesiologists physical status; WBC, white blood cell; VAS, visual analogue scale.

lar diet $(\mathrm{P}<0.001)$ and postoperative hospital stay $(\mathrm{P}<0.001)$ were found between the 2 groups. The mean operation time was longer in the traditional group than in the PCN group $(\mathrm{P}=0.003)$. Age, sex, BMI, underlying disease, ASA physical status classification, and other clinicopathological outcomes showed no statistical differences. No postoperative morbidity, including surgical site infection, postoperative mortality, or reoperation within 30 days of surgery, was noted.

Multivariate analyses using linear regression showed that traditional nutrition, i.e., an advance to full liquid or soft diet and then regular diet as tolerated, was the only factor associated with postoperative hospital stay (Table 2).

Outcomes stratified by length of postoperative hospital stay (LOS) are shown in Table 3. LOS was 3 days or longer in 73 patients, and a statistically significant difference in LOS according to the type of nutrition was found $(\mathrm{P}<0.001)$. Time to tolerance of regular diet was longer in patients with LOS $\geq 3$ days $(\mathrm{P}<0.001)$.
Table 2. Factors associated with postoperative hospital stay, as determined by using multivariate analyses with linear regression

\begin{tabular}{lcc}
\hline Variable & Regression coefficients & P-value \\
\hline Traditional nutrition & 1.016 & $<0.001$ \\
Age $(\mathrm{yr})$ & 0.008 & 0.093 \\
Male sex & 0.139 & 0.394 \\
Body mass index $\left(\mathrm{kg} / \mathrm{m}^{2}\right)$ & -0.012 & 0.624 \\
Operation time $(\mathrm{min})$ & -0.001 & 0.918 \\
Preoperative WBC count $\left(/ \mathrm{mm}^{3}\right)$ & $\mathrm{N} / \mathrm{A}$ & $\mathrm{N} / \mathrm{A}$ \\
Pathology, serosal inflammation & & \\
$\quad$ Mild & -0.052 & 0.834 \\
Severe & -0.048 & 0.849 \\
\hline
\end{tabular}

WBC, white blood cell; N/A, not available.

Table 3. Clinicopathological characteristics stratified by length of postoperative hospital stay (LOS)

\begin{tabular}{|c|c|c|c|}
\hline Characteristics & $\begin{aligned} \mathrm{LOS} & \leq 2 \text { days } \\
(\mathrm{n} & =106)\end{aligned}$ & $\begin{array}{c}\mathrm{LOS} \geq 3 \text { days } \\
(\mathrm{n}=73)\end{array}$ & P-value \\
\hline $\begin{array}{l}\text { Methods of postoperative } \\
\text { nutrition }\end{array}$ & & & $<0.001$ \\
\hline Patient-controlled nutrition & $72(67.9)$ & $12(16.4)$ & \\
\hline Traditional nutrition & $34(32.1)$ & $61(83.6)$ & \\
\hline Age (yr) & $30.9 \pm 14.7$ & $36.8 \pm 19.5$ & 0.031 \\
\hline Sex, male : female & $44: 62$ & $41: 32$ & 0.054 \\
\hline \multicolumn{4}{|l|}{ Body mass index $\left(\mathrm{kg} / \mathrm{m}^{2}\right)$} \\
\hline Underlying disease (\%) & $15(14.2)$ & $10(13.7)$ & 1.000 \\
\hline ASA PS classification & & & 1.000 \\
\hline । & $101(95.3)$ & $69(94.5)$ & \\
\hline$\|$ & $4(3.8)$ & $3(4.1)$ & \\
\hline III & $1(0.9)$ & $1(1.4)$ & \\
\hline IV & $0(0)$ & $0(0)$ & \\
\hline Preoperative WBC count $\left(/ \mathrm{mm}^{3}\right)$ & $12,189 \pm 4,638$ & $12,763 \pm 4,649$ & 0.418 \\
\hline $\begin{array}{l}\text { History of previous abdominal } \\
\text { surgery }\end{array}$ & $12(11.3)$ & $6(8.2)$ & 0.498 \\
\hline $\begin{array}{l}\text { Postoperative pain within } 24 \\
\text { hours (VAS) }\end{array}$ & $6.3 \pm 1.1$ & $6.4 \pm 0.83$ & 0.373 \\
\hline Postoperative opioid use & $44(41.5)$ & $36(49.3)$ & 0.302 \\
\hline Pathology, serosal inflammation & & & 0.757 \\
\hline No & $14(13.2)$ & $7(9.6)$ & \\
\hline Mild & $44(41.5)$ & $31(42.5)$ & \\
\hline Severe & $48(45.3)$ & $35(47.9)$ & \\
\hline Operation time (min) & $33.6 \pm 13.0$ & $39.5 \pm 17.1$ & 0.010 \\
\hline $\begin{array}{l}\text { Time to tolerance of regular diet } \\
\text { (hr) }\end{array}$ & $9.6 \pm 7.5$ & $16.2 \pm 5.6$ & $<0.001$ \\
\hline
\end{tabular}

Values are presented as number (\%) or mean \pm standard deviation.

WBC, white blood cell; VAS, visual analog scale. 
Table 4. Factors associated with prolonged postoperative hospital stay ( $\geq 3$ days), as determined by using multivariate analyses with logistic regression

\begin{tabular}{lccc}
\hline Characteristic & Odds ratio & $\begin{array}{c}95 \% \text { Confidence } \\
\text { interval }\end{array}$ & P-value \\
\hline Traditional nutrition & 9.409 & $4.311-20.540$ & $<0.001$ \\
Age $(\mathrm{yr})$ & 1.015 & $0.992-1.038$ & 0.216 \\
Male sex & 1.196 & $0.538-2.659$ & 0.660 \\
Body mass index (kg/m²) & 1.025 & $0.907-1.159$ & 0.691 \\
Operation time (min) & 1.007 & $0.983-1.032$ & 0.569 \\
Preoperative WBC count $\left(/ \mathrm{mm}^{3}\right)$ & 1.000 & $1.000-1.000$ & 0.895 \\
Pathology, serosal inflammation & & & \\
$\quad$ Mild & 1.509 & $0.417-5.458$ & 0.530 \\
$\quad$ Severe & 1.155 & $0.309-4.313$ & 0.830 \\
\hline
\end{tabular}

WBC, white blood cell.

Multivariate analyses using logistic regression showed that traditional nutrition was the only risk factor associated with prolonged postoperative hospital stay ( $\geq 3$ days) (Table 4 ).

\section{DISCUSSION}

Usually, once patients demonstrate tolerance of a clear liquid diet after abdominal surgery, the diet is advanced to a full liquid diet, a soft diet, and then a regular diet, as tolerated. In this widespread practice, PCN is a novel concept in postoperative nutrition after abdominal surgery. The present study shows the feasibility of PCN after abdominal surgery, especially minimally invasive surgery.

A recently launched postoperative management method called Fast Track or Early Recovery after Surgery (ERAS) includes EOF. Especially in minimally invasive abdominal surgery, EOF has become usual practice to accelerate patient recovery $[5,6]$. However, few studies have looked closely at the initial meals after surgery regardless of early or delayed oral feeding. A prospective randomized study regarding type of initial diet was performed in patients undergoing GI operations, and it showed no significant difference between the groups given a clear liquid or solid diet soon after removal of a nasogastric tube [9]. Another randomized controlled trial in gynecologic oncology patients undergoing intraabdominal surgery showed that a regular diet as the first meal after abdominal surgery was safe and efficacious [11].

A deep-seated, yet unfounded, concern about the risk of increasing postoperative nausea and vomiting, as well as anxiety about unlikely prolonged postoperative ileus slowing the advancement of postoperative diet unnecessarily, is still prevalent. Moreover, a delayed diet requires additional fasting for the patients, often to the patient's chagrin. Furthermore, liquid and soft diets are rather unpalatable, and patients are often very receptive to a regular diet soon after surgery. In actuality, a patient's appetite may be a good indicator for postoperative feeding [12]. The most reliable indicator of tolerability to postoperative oral feeding is currently the subjective condition of the patients themselves. In practice, patients usually want to choose the time and type of diet themselves, as long as surgical complications that require fasting do not occur. Most patients intuitively know whether they are ready to eat and what would be tolerated. In one study, with this individualized and patient-oriented concept, solid meals were safely initiated by the second operative day in $81.3 \%$ of patients who had undergone elective colon cancer surgery [12]. Another previous survey showed that patients preferred solid foods as early as the first postoperative day after colorectal surgery and that their preferences had little congruency with the traditional clear liquid diet [13].

In the present study, patients who could select their diet depending on comfort level (PCN group) were tolerable to regular diet $5.5 \pm 3.9$ hours after surgery on average whereas the traditional group took $18.3 \pm 3.8$ hours. Korean patients are usually discharged from hospital to home after they are able to tolerate a regular diet, so delayed advancement of the diet may often prolong postoperative hospital stay. The important thing is that this delay of diet advancement in the traditional group was likely unnecessary.

According to a well-designed prospective study on a laparoscopic versus an open appendectomy, postoperative reintroduction of nutrition followed strict criteria: bowel sounds were checked every 12 hours, a clear liquid diet was started after bowel sounds were present, and advancement to a regular diet occurred when the liquid diet was tolerated and flatus was observed [14]. Although general anesthesia could contribute to postoperative ileus [15], evidence is amounting that a delay in solid foods, in contrast to patient comfort, is often unnecessary.

The purpose of a clear liquid diet is to leave no residue in the intestinal tract and to provide oral hydration during acute illness or before and after surgery [16]. However, a regular diet has been shown to neither interfere with oral hydration nor promote dietary intolerance [17]. For the conventional postoperative diet, no significant evidence supporting its benefit in regard to borborygmus, abdominal distension or pain, or nausea or vomiting is available. Interpretation of bowel function based on bowel sounds, flatulence, or bowel movement, which are surrogate markers of bowel motility, after abdominal surgery does not correlate with bowel functional status [8]. However, the conventional conservative approach to postoperative feeding has been maintained by most surgeons who regard "as tolerated" to be an important factor in diet progression.

The conventional postoperative diet may be an unfounded surgical dogma, as was the past routine use of nasogastric tubes to decompress the GI tract after abdominal surgery. However, evidence that nasogastric tubes are not beneficial after abdominal surgery is now available; furthermore, more than half a century was required to garner sufficient evidence to support changing 
that surgical dogma $[1,2]$.

The ERAS program in colonic surgery includes 17 fast track (FT) elements: preoperative counseling, preoperative feeding, synbiotics, no bowel preparation, no premedication, fluid restriction, perioperative high oxygen concentrations, active prevention of hypothermia, epidural analgesia, minimally invasive/transverse incisions, no routine use of nasogastric tubes, no use of drains, enforced postoperative mobilization, enforced postoperative oral feeding, no systemic opioid use, use of standard laxatives, and early removal of the bladder catheter [18]. At least four FT elements should be chosen for implementation of the ERAS program. All studies regarding the ERAS program included enforced postoperative oral feeding and enforced postoperative mobilization [18]. Indeed, enforced postoperative oral feeding could be one of the most important elements of the ERAS program. Time to tolerance of a regular diet is closely related with methods to advance the diet. Our PCN concept may reduce the time to tolerance of a regular diet and shorten postoperative hospital stay.

This was a pilot study conducted with laparoscopic appendectomy patients who had simple appendicitis to evaluate the feasibility and effectiveness of PCN, as well as its effect on postoperative outcomes, following abdominal surgery. Our data show that a traditional postoperative diet might be a surgical dogma based on superstitious concern and anxiety. After a laparoscopic appendectomy for simple appendicitis, 'method of postoperative nutrition' was the only clinicopathologic variable that influenced postoperative hospital stay.

The present study had no postoperative morbidity, including surgical site infection, postoperative mortality, or reoperation within 30 days of the surgery. The laparoscopic appendectomy is one of the simplest minimally invasive abdominal surgeries; patients with complicated (perforated, gangrenous, or with abscess) appendicitis was excluded from this study. The application of $\mathrm{PCN}$ in patients who undergo elective laparoscopic colorectal surgery will be the focus of a future study.

In conclusion, after abdominal surgery, especially minimally invasive surgery, PCN may be a feasible and effective novel concept in postoperative nutrition. In the ERAS program, our PCN concept may reduce the time to tolerance of a regular diet and shorten postoperative hospital stay. Wide application of PCN is recommended in patients who undergo elective minimally invasive surgery.

\section{CONFLICT OF INTEREST}

No potential conflict of interest relevant to this article was reported.

\section{REFERENCES}

1. Cheatham ML, Chapman WC, Key SP, Sawyers JL. A meta-analysis of selective versus routine nasogastric decompression after elective laparotomy. Ann Surg 1995;221:469-76.

2. Nelson R, Tse B, Edwards S. Systematic review of prophylactic nasogastric decompression after abdominal operations. Br J Surg 2005;92:673-80.

3. da Fonseca LM, Profeta da Luz MM, Lacerda-Filho A, Correia MI, Gomes da Silva R. A simplified rehabilitation program for patients undergoing elective colonic surgery--randomized controlled clinical trial. Int J Colorectal Dis 2011;26:609-16.

4. El Nakeeb A, Fikry A, El Metwally T, Fouda E, Youssef M, Ghazy $\mathrm{H}$, et al. Early oral feeding in patients undergoing elective colonic anastomosis. Int J Surg 2009;7:206-9.

5. Lee TG, Kang SB, Kim DW, Hong S, Heo SC, Park KJ. Comparison of early mobilization and diet rehabilitation program with conventional care after laparoscopic colon surgery: a prospective randomized controlled trial. Dis Colon Rectum 2011;54:21-8.

6. Kim HO, Lee SR, Choi WJ, Kim H. Early oral feeding following laparoscopic colorectal cancer surgery. ANZ J Surg 2014;84:53944.

7. Warren J, Bhalla V, Cresci G. Postoperative diet advancement: surgical dogma vs evidence-based medicine. Nutr Clin Pract 2011;26:115-25.

8. Schippers E, Hölscher AH, Bollschweiler E, Siewert JR. Return of interdigestive motor complex after abdominal surgery. End of postoperative ileus? Dig Dis Sci 1991;36:621-6.

9. Bickel A, Shtamler B, Mizrahi S. Early oral feeding following removal of nasogastric tube in gastrointestinal operations. A randomized prospective study. Arch Surg 1992;127:287-9.

10. Kim HO, Yoo CH, Lee SR, Son BH, Park YL, Shin JH, et al. Pain after laparoscopic appendectomy: a comparison of transumbilical single-port and conventional laparoscopic surgery. J Korean Surg Soc 2012;82:172-8.

11. Pearl ML, Frandina M, Mahler L, Valea FA, DiSilvestro PA, Chalas E. A randomized controlled trial of a regular diet as the first meal in gynecologic oncology patients undergoing intraabdominal surgery. Obstet Gynecol 2002;100:230-4.

12. Kawamura YJ, Kuwahara Y, Mizokami K, Sasaki J, Tan KY, Tsujinaka $S$, et al. Patient's appetite is a good indicator for postoperative feeding: a proposal for individualized postoperative feeding after surgery for colon cancer. Int J Colorectal Dis 2010;25:23943.

13. Yeung SE, Fenton TR. Colorectal surgery patients prefer simple solid foods to clear fluids as the first postoperative meal. Dis Colon Rectum 2009;52:1616-23.

14. Katkhouda N, Mason RJ, Towfigh S, Gevorgyan A, Essani R. Laparoscopic versus open appendectomy: a prospective randomized double-blind study. Ann Surg 2005;242:439-48.

15. Larsson S, Lundberg D. A prospective survey of postoperative nausea and vomiting with special regard to incidence and relations to patient characteristics, anesthetic routines and surgical procedures. Acta Anaesthesiol Scand 1995;39:539-45.

16. Hancock S, Cresci G, Martindale R. The clear liquid diet: when is it appropriate? Curr Gastroenterol Rep 2002;4:324-31. 


\section{Coloproctology Hyung Ook Kim, et al.}

17. Jeffery KM, Harkins B, Cresci GA, Martindale RG. The clear liquid diet is no longer a necessity in the routine postoperative management of surgical patients. Am Surg 1996;62:167-70.
18. Wind J, Polle SW, Fung Kon Jin PH, Dejong CH, von Meyenfeldt MF, Ubbink DT, et al. Systematic review of enhanced recovery programmes in colonic surgery. Br J Surg 2006;93:800-9. 